\section{Neue Antibiotika zur Therapie von Atemwegsinfektionen}

\author{
New Antibiotics for the Therapy of Respiratory Tract Infections
}

\section{Zusammenfassung}

Der Bedarf an neuen Antibiotika ist aufgrund vielfältiger Resistenzentwicklungen groß. Die Resistenz bei Streptococcus pneumoniae gegenüber Makroliden und Penicillinen wird in den USA bereits auf über $30 \%$ beziffert. Die Zulassung neuer Antibiotika, insbesondere solche mit neuem Wirkmechanismus, ist seit vielen Jahren rückläufig, so dass in Zukunft mit einem Mangel an wirksamen Therapieoptionen gerechnet werden muss. Neue Substanzen mit guter In-vitro-Aktivität gegenüber Pneumokokken haben sich nicht in allen Fällen als klinisch unbedenklich erwiesen, wie es zum Beispiel für mehrere Chinolone zutrifft. Ebenso konnte Daptomycin, ein Lipopeptid mit neuartigem Wirkprinzip, aufgrund der Deaktivierung durch Surfactant bei Atemwegserkrankungen nicht überzeugen. Etabliert haben sich bei den Chinolonen Levofloxacin und Moxifloxacin, das seit 2002 auch als i.v.-Zubereitung zur Verfügung steht. Bei den Ketoliden sind Telithromycin (nur oral) und bei den Azaliden Azithromycin (seit 2005 auch als i.v.-Zubereitung) im Handel. Linezolid ist das bisher einzige zugelassene Oxazolidinon. Im Laufe des Jahres 2006 ist in Deutschland mit der Zulassung eines neuen Tetracyclinderivats (Tigecyclin) zu rechnen. Als neues Carbapenemen wurde Ertapenem im Jahr 2003 zugelassen, ein weiteres Antibiotikum aus dieser Gruppe, Doripenem, befindet sich noch in der klinischen Entwicklung.

\section{Abstract}

The need for new antibiotics is ongoing in view of manifold resistance developments. The resistance of Streptococcus pneumoniae against macrolides and penicillins in the USA already amounts to more than $30 \%$. The approval of new antibiotics, particularly those with a new mode of action, has been decreasing over the past years which is anticipated to result in a lack of resourceful therapy options in the future. Some new substances with good in vitro activity against S. pneumoniae have turned out to raise safety issues as shown for a number of quinolones. Likewise daptomycin, a lipopeptide with a new mechanism of action, did not show satisfying results due to its inactivation by surfactant. Established quinolones are levofloxacin and moxifloxacin, the latter being available as i.v. formulation since 2002. For ketolides, telithromycin (oral) and for azalides azithromycin (since 2005 as i.v. formulation) are available for antimicrobial therapy. Linezolid is the only approved oxazolidinone. Tigecycline, a tetracycline derivate, will be approved in 2006 in Germany. The latest approval regarding carbapenems was ertapenem in 2003, while doripenem, a new antibiotic from this group, is still under clinical development. 
Mehr als 70 Jahre nach der Entdeckung von Penicillin durch Alexander Fleming besteht nach wie vor ein Bedarf an neuen Antibiotika. In erster Linie sind es die vielfältigen Resistenzentwicklungen, die im Wettlauf mit den Erregern den Einsatz neuer, wirksamer Antibiotika notwendig machen. Besorgniserregend ist zum Beispiel die weltweit zunehmende Resistenzentwicklung auch bei Streptococcus pneumoniae - dem führenden Erreger bakterieller Infektionen der Atemwege. In den USA sind bereits 30\% der Pneumokokken resistent gegenüber Makroliden, die Häufigkeit der Penicillinresistenz liegt sogar noch höher.

Die Bereitschaft der pharmazeutischen Hersteller in neue antimikrobiell wirksame Arzneimittel zu investieren, ist seit Jahren rückläufig, so dass in Zukunft mit einem zunehmenden Mangel an hochwirksamen Antiinfektiva gerechnet werden muss (Abb.1) [1]. Diese durchaus bedrohliche Situation hat die USamerikanische infektiologische Gesellschaft (IDSA) bereits im Jahre 2003 dazu bewogen, eine spezielle Arbeitsgruppe zu bilden und die Situation zu analysieren. Als eine Ursache der misslichen Situation wird der relativ geringe finanzielle Gewinn genannt, der im Vergleich mit anderen Arzneimitteln mit dem Vertrieb von Antibiotika verbunden ist. Die Auswertung der Arbeitsgruppe gipfelte in der Aussage „Bad bugs, no drugs ...." Mit „bad bugs“ sind dabei vor allem die sechs in der Tab. 1 genannten Erreger gemeint, die bei der stationären Versorgung der Patienten zunehmend Probleme bereiten [2]. Angesichts der therapeutischen Probleme, die zum Beispiel multiresistente A. baumannii Stämme verursachen, nahm in den vergangenen Jahren sogar die Bedeutung von Colistin, einem lang bekannten Antibiotikum wieder zu. Colistin ist häufig die einzige wirksame Substanz gegen A. baumannii. In Deutschland ist es nicht zur systemischen Therapie im Handel, die Anwendung gilt aufgrund der toxikologischen Risiken als obsolet. Die einzige neue mögliche Therapieoption bei diesem Erreger besteht mit Tigecyclin, das im Laufe des Jahres 2006 in Europa zugelassen werden soll (s. unten). Dieses und weitere Antibiotika, die kurz vor der Zulassung stehen, sollen weiter unten kurz beschrieben werden.

Zunächst werden hier jedoch die wenigen Substanzen diskutiert, die in den vergangenen Jahren in Deutschland zugelassen wurden. Einen Überblick gibt die Tab. 2. Seit 2001 kamen lediglich

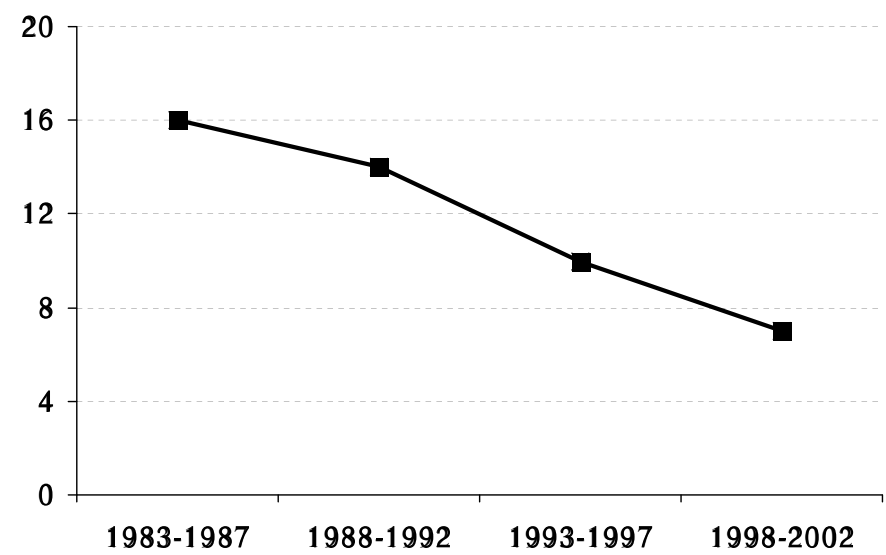

Abb. 1 Anzahl der neuen Arzneimittel zur antimikrobiellen Therapie, die zwischen 1983 und 2002 in den USA zugelassen wurden (mod. nach [1]).
Tab 1 „Bad bugs“ - Erreger, die zunehmend therapeutische Probleme bereiten

1. Acinetobacter baumannii

2. Aspergillus Spezies

3. Enterobacteriaceae*

4. Enterococcus faecium (Vancomycin-resistent)

5. Pseudomonas aeruginosa

6. Staphylococcus aureus (Methicillin-resistent, MRSA)

* Stämme mit Bildung von $\beta$-Laktamasen mit erweitertem Spektrum

( = extended spectrum $\beta$-lactamase (ESBL)-producing strains, mod. n. [2]).

vier neue Medikamente zur antibakteriellen Therapie auf den Markt: Ketek ${ }^{\circledR}$ (Telithromycin), Zyvoxid ${ }^{\circledR}$ (Linezolid) und Bonoq $^{\circledR}$ (Gatifloxacin; inzwischen wieder aus dem Handel genommen) sowie Invanz ${ }^{\circledR}$ (Ertapenem). Darüber hinaus wurden in diesem Zeitraum neue Zubereitungsformen von bereits früher eingeführten Substanzen in den Handel gebracht, die zu einer besseren Verträglichkeit beitragen oder die antimikrobielle Therapie optimieren sollen. Dies trifft z. B. für die i. v.-Zubereitungen von Moxifloxacin (Avalox ${ }^{\circledR} 400$ Infusionslösung) oder Azithromycin (Zithromax ${ }^{\circledR}$ i.v.) zu, die unten ausführlicher beschrieben werden.

\section{Chinolone: neue Derivate nur in den USA im Handel}

Ein Blick auf die Entwicklung der letzten Jahre zeigt, dass nicht alle neuen Antiinfektiva die in sie gesetzten Erwartungen erfüllen konnten. So sind etliche Chinolone mit guter In-vitro-Aktivität gegen Pneumokokken - wie Clinafloxacin, Sparfloxacin, Grepafloxacin oder Trovafloxacin - während der klinischen Entwicklung gestoppt worden oder kurz nach der Markteinführung wieder zurückgezogen worden. Bei den drei zuerst genannten lagen die Gründe hierfür in einer ausgeprägten Phototoxizität bzw. einem relativ hohen kardiotoxischen Potenzial. Trovafloxacin wird aufgrund von sehr selten auftretenden, aber schwerwiegenden hepatotoxischen Effekten nicht mehr angewandt [3].

Weitere Chinolone, die sich bei Atemwegsinfektionen als gut wirksam erwiesen haben, sind Gatifloxacin und Gemifloxacin. Beide besitzen eine gute in vitro Aktivität gegen Pneumokokken, wie sich an den MHK-Werten ablesen lässt (Tab.3). Sie sind in Deutschland nicht im Handel, werden aber zum Beispiel in den USA bei bakteriellen Infektionen der unteren Atemwege häufig angewandt. Mit beiden Substanzen können Nebenwirkungen auftreten, die bei der Therapie mit anderen Chinolonen nicht vorkommen bzw. sehr viel seltener sind. Gatifloxacin wird in den USA unter dem Handelsnamen Tequin ${ }^{\circledR}$ von Bristol-Myers Squibb vertrieben. Es verursacht häufiger als andere Chinolone Störungen im Glukosestoffwechsel. In Deutschland war es unter dem Namen Bonoq $^{\circledR}$ zwischen 2001 und 2003 verfügbar. Im Rahmen der Zulassungsbemühungen des Herstellers für alle europäischen Staaten wurden von der EMEA in London die vereinzelt aufgetretenen hypo- und hyperglykämischen Unverträglichkeitsreaktionen dieser Substanz neu bewertet und das Nutzen-Risiko-Verhältnis wurde anders als von der FDA eingestuft. Die europäischen Zulassungsbehörden reduzierten daher den Anwendungsbereich auf die beiden Indikationen „ambulant erworbene Pneumonie“ und 
Tab. 2 Übersicht über die in diesem Beitrag besprochenen Antibiotika

\begin{tabular}{|c|c|c|c|c|}
\hline Gruppe & Freiname & Handelsname & $\begin{array}{l}\text { in Deutschland im Handel } \\
\text { seit (ca.) }\end{array}$ & Bemerkung \\
\hline \multirow[t]{5}{*}{ Chinolone } & Moxifloxacin & Avalox ${ }^{1}$ Infusionslösung & 2002 (i.v.-Zubereitung) & orale Zubereitung seit 1999 im Handel \\
\hline & Levofloxacin & Tavanic & 1998 & Enantiomer von Ofloxacin \\
\hline & Gatifloxacin & Tequin (USA) & - & seit 2003 nicht mehr im Handel (Bonoq) \\
\hline & Gemifloxacin & Factive (USA) & - & häufige UAW: Exantheme \\
\hline & Garenoxacin & - & - & in klinischer Prüfung \\
\hline \multirow[t]{2}{*}{ Ketolide } & Telithromycin & Ketek & 2001 & Makroliderivat \\
\hline & Cethromycin & - & - & klinische Entwicklung gestoppt \\
\hline Azalide & Azithromycin & Zithromax i.v. & 2005 (i.v.-Zubereitung) & orale Zubereitung seit 1993 im Handel \\
\hline Oxazolidinone & Linezolid & Zyvoxid & 2001 & Blutbildveränderungen beachten \\
\hline \multirow[t]{2}{*}{ Carbapeneme } & Ertapenem & Invanz & 2003 & nur zur parenteralen Gabe \\
\hline & Doripenem & - & - & in klinischer Prüfung \\
\hline Lipopeptide & Daptomycin & Cubicin (USA) & - & Inaktivierung durch Surfactant \\
\hline Glycylcycline & Tigecyclin & Tygacil (USA) & - & europaweite Zulassung in 2006 erwartet \\
\hline
\end{tabular}

1 in USA Avelox

„komplizierte Harnwegsinfektionen“. Wegen dieser erheblichen Einschränkungen der Indikationsgebiete entschloss sich die verantwortliche pharmazeutische Firma Grünenthal (Aachen) die Substanz vom europäischen Markt zu nehmen.

Gemifloxacin ist in den USA unter dem Namen Factive ${ }^{\circledR}$ verfügbar, es gehört zu den Chinolonen mit der höchsten Pneumokokken-Aktivität und wird von der Firma Oscient bei „ambulant erworbener Pneumonie“ und „akuter Exazerbation einer chronischen Bronchitis“ angeboten. Das Chinolon wurde ursprünglich in Korea entwickelt, die klinische Prüfung wurde von Smith Kline Beecham, später GlaxoSmithKline, durchgeführt. Die Substanz wurde von der FDA zunächst nicht zugelassen, weil Unklarheiten hinsichtlich der Verträglichkeit bestanden. Gemifloxacin führt bei Frauen vor der Menopause sehr häufig zu Exanthemen. Die Inzidenz der Hautreaktionen steigt mit der Therapiedauer und betrifft bei Frauen unter 40 Jahren und einer Therapie über 14 Tage etwa jede 4. Frau. Bei Männern, postmenopausalen Frauen und vor allem bei kurzer Behandlungsdauer von weniger als 5 Tagen liegt die Exanthemrate bei unter 3\%. In Deutschland ist es nicht im Handel.

Weitere Chinolone mit guter Wirksamkeit bei PneumokokkenInfektionen finden sich zurzeit in unterschiedlichen Phasen der klinischen Entwicklung. Dabei ist bemerkenswert, dass einige der neueren Derivate nicht in Position 6 des Grundgerüstes fluoriert sind. Die Bezeichnung der Wirkstoffgruppe als „Fluorchinolone“ muss daher als nicht mehr zutreffend angesehen werden, wenn die Neuentwicklungen mit berücksichtigt werden sollen. Der Name „Chinolone“ ist unter diesen Aspekten geeigneter. $\mathrm{Zu}$ diesen Substanzen gehört Garenoxacin, das sich seit einigen Jahren in der klinischen Prüfung befindet. Ursprünglich von der japanischen Firma Toyama entwickelt, wurde die klinische Prüfung der Substanz von Bristol-Myers Squibb durchgeführt, seit 2004 besitzt die amerikanische Firma Schering-Plough die Rechte für die weltweite Vermarktung des Chinolons. Die Substanz zeichnet sich durch ein breites Spektrum aus, besitzt eine hohe Aktivität gegen Pneumokokken und erfasst auch anaerobe Bakterien.

\section{Bewährte Chinolone in neuer Zubereitungsform}

Unter den etablierten Vertretern der Chinolone hat Levofloxacin aufgrund seiner deutlich höheren antibakteriellen Aktivität Ofloxacin ersetzt. Chemisch gesehen stellt der Wirkstoff Ofloxacin ein Gemisch (Racemat) aus zwei optischen Enantiomeren dar: einer R- und einer S-Form. Da nur eine dieser beiden unterschiedlichen Verbindungen antibakteriell wirksam ist, lag es nahe, den eigentlichen Wirkstoff zur Therapie anzubieten. Die S-Form aus dem Racemat Ofloxacin wird jetzt als Levofloxacin bevorzugt zur Therapie eingesetzt. Da Levofloxacin etwa doppelt so wirksam ist wie Ofloxacin und zudem höher dosiert wird, werden Pneumokokken und andere grampositive Erreger als empfindlich eingestuft. Damit ist Levofloxacin bei bakteriellen Atemwegsinfektionen anwendbar. Levofloxacin wird nach oraler Gabe praktisch vollständig aus dem Magen-Darmtrakt resorbiert. Die maximale Plasmakonzentration nach Einnahme von $500 \mathrm{mg}$ liegt bei etwa $5 \mathrm{mg} / \mathrm{l}$, etwas höhere Werte ergeben sich nach intravenöser Verabreichung $(6,3 \mathrm{mg} / \mathrm{l})$. Die Eliminationshalbwertzeit beträgt etwa $6-8$ Stunden. Die Elimination von Levofloxacin erfolgt fast ausschließlich renal und ist daher von der Nierenfunktion abhängig. Bei einer Kreatinin-Clearance von 20 bis $50 \mathrm{ml} / \mathrm{min}$ beträgt die Eliminationshalbwertzeit zum Beispiel etwa 27 Stunden. Diese Zusammenhänge müssen berücksichtigt werden, um eine Kumulation zu vermeiden. Prinzipiell ist zu bedenken, dass bei jedem älteren Patienten mit einer physiologischen Einschränkung der renalen Elimination zu rechnen ist.

Moxifloxacin weist von allen in Deutschland verfügbaren Chinolonen die höchste Pneumokokken-Aktivität auf (Tab.3). Es hat sich als gut verträgliches und wirksames Arzneimittel in den vergangenen Jahren bewährt. Als Infusionszubereitung wurde Moxifloxacin erst einige Jahre nach der Tablettenform eingeführt. Damit ist eine rationale Therapie schwerkranker Patienten mit ambulant erworbener Pneumonie möglich. Obwohl die Substanz praktisch vollständig aus dem Magen-Darmtrakt resorbiert wird, ist die Verfügbarkeit der Zubereitung zur intravenösen Applikation begrüßenswert, weil schwerstkranke Patienten gelegentlich 
Tab. 3 Antibakterielle Aktivität von Chinolonen*

\begin{tabular}{|c|c|c|c|c|c|c|}
\hline \multirow[t]{2}{*}{ Erreger } & \multicolumn{6}{|l|}{ Chinolon } \\
\hline & Ciprofloxacin & Levofloxacin & Gatifloxacin $^{a}$ & Moxifloxacin & Gemifloxacin ${ }^{a}$ & Garenoxacin $^{b}$ \\
\hline Haemophilus influenzae & 0,016 & 0,06 & 0,013 & 0,06 & 0,03 & 0,03 \\
\hline Moraxella catarrhalis & 0,03 & 0,03 & $\leq 0,6$ & 0,03 & 0,015 & 0,03 \\
\hline Pseudomonas aeruginosa & 4 & 32 & 32 & 8 & $\geq 8$ & $>8$ \\
\hline Chlamydia pneumoniae & $\leq 2,0$ & $\leq 0,5$ & $\leq 0,125$ & $\leq 1$ & 0,25 & 0,015 \\
\hline
\end{tabular}

* $\mathrm{MHK}_{90}$-Werte, minimale Hemmkonzentrationen ( $\left.\mathrm{mg} / \mathrm{l}\right)$, bei der mindestens $90 \%$ der untersuchten Stämme gehemmt werden. Die angegebenen MHK-Werte wurden aus aktuellen Publikationen entnommen; sie erlauben einen Vergleich der antibakteriellen in vitro Aktivität der einzelnen Derivate. Es muss beachtet werden, dass sie regional sehr unterschiedlich sein können und aufgrund der Resistenzentwicklung in unterschiedlichem Ausmaß je nach Art des Erregers zeitlichen Veränderungen unterliegen.

a = Gatifloxacin und Gemifloxacin sind in Deutschland nicht im Handel.

$\mathrm{b}=$ Garenoxacin ist ein in Position 6 des Grundgerüstes nicht fluoriertes Derivat. Es befindet sich derzeit in der klinischen Entwicklung

(mod. nach [42] und anderen Quellen).

veränderte Resorptionsverhältnisse aufweisen, zum Erbrechen neigen oder aus anderen Gründen nicht oral behandelt werden können.

Sowohl Penicillin-sensible als auch Penicillin-resistente Stämme von S. pneumoniae werden bereits durch Konzentrationen von 0,125 - 0,25 mg Moxifloxacin/l gehemmt (Tab. 3). Darüber hinaus erfasst Moxifloxacin auch andere klinisch bedeutsame Erreger von Atemwegsinfektionen, einschließlich der so genannten atypischen Erreger. Am Ende einer einmaligen intravenösen Infusion über 60 Minuten wurden Spitzenkonzentrationen von 4,1 mg/l erreicht (zum Vergleich: 3,1 mg/l nach oraler Gabe). Die AUC-Werte, die nach oraler $(35 \mathrm{mg} / \mathrm{l} \times \mathrm{h})$ und intravenöser $(39 \mathrm{mg} / \mathrm{l} \times \mathrm{h})$ Gabe ermittelt wurden, unterscheiden sich aufgrund der guten Bioverfügbarkeit nur minimal. Zu den Vorteilen der Chinolone gehört ein relativ hohes scheinbares Verteilungsvolumen. Die Substanz wird nicht durch Monooxygenasen metabolisiert, sondern in Form von zwei Phase-II-Metaboliten ausgeschieden (als N-Sulfo-Metabolit und Glucuronid). Die Eliminationshalbwertzeit liegt bei 11 bis 12 Stunden, bei eingeschränkter Nierenfunktion ist die Kinetik nicht verändert und die Dosierung muss nicht reduziert werden; dies gilt auch für dialysepflichtige Patienten.

Die Infusionslösung mit 400 mg Wirkstoff ist zur Behandlung der ambulant erworbenen Pneumonie zugelassen. Die empfohlene Infusionsdauer von 60 Minuten sollte nicht unterschritten werden. Bei entsprechender klinischer Situation kann die Behandlung mit der Tablettenzubereitung fortgeführt werden.

\section{Klinische Studien mit Chinolonen bei Pneumonie}

In zwei umfangreichen klinischen Studien, von denen eine doppelblind durchgeführt wurde, ist die Wirksamkeit der Sequenzialtherapie mit einer einmal täglichen Gabe belegt worden. Die klinischen Erfolgsraten lagen in beiden Studien bei 84-86\% der behandelten Patienten; die Beurteilung erfolgte bis zu vier Wochen nach der Behandlung. Als Vergleichssubstanzen dienten andere Chinolone (Levofloxacin bzw. Trovafloxacin) oder Coamoxiclav, das je nach klinischer Situation mit oder ohne zusätzliches Makrolid gegeben wurde. Während die Rate des klinischen Erfolgs bei den mit anderen Chinolonen behandelten Patienten keinen Unterschied zum Erfolg bei den Moxifloxacin-behandelten Patienten aufwies, war die Behandlung mit Coamoxiclav statistisch signifikant weniger wirksam (74\% Erfolg 3-4 Wochen nach Abschluss der Therapie) [4,5].

In aktuellen Richtlinien werden Moxifloxacin und Levofloxacin als gleichwertige Alternativen zu der Therapie mit einem $\beta$-Laktamantibiotikum in Kombination mit einem Makrolid empfohlen. In den USA wurde eine groß angelegte Doppelblindstudie bei fast 400 Patienten mit einem mittleren Lebensalter von 77 Jahren durchgeführt. Die Studie war von der US-amerikanischen Zulassungsbehörde FDA gefordert worden, um vergleichende Daten über die Wirksamkeit und Verträglichkeit der beiden Chinolone zu erstellen. Jeweils die Hälfte der Patienten erhielt entweder Moxifloxacin $(1 \times \operatorname{tgl} .400 \mathrm{mg})$ oder Levofloxacin $(1 \times \mathrm{tgl}$. $500 \mathrm{mg}$ ) für 7 bis 14 Tage. Bei Patienten mit eingeschränkter Nierenfunktion wurde die Dosierung von Levofloxacin auf $250 \mathrm{mg}$ reduziert. Beide Präparate wurden für mindestens 2 Tage intravenös verabreicht, anschließend wurde bei entsprechendem klinischen Zustand des Patienten die Therapie mit Tabletten fortgesetzt. Fünf bis 21 Tage nach Abschluss der Therapie zeigte sich ein Therapieerfolg bei etwa $90 \%$ der Patienten in beiden Gruppen (Abb. 2). Beide Chinolone waren etwa gleich gut verträglich. Die Behandlung wurde bei 7,7\% (Moxifloxacin) bzw. 10,1\% (Levofloxacin) der Patienten wegen unerwünschter Wirkungen abgebrochen. Am häufigsten waren gastrointestinale Störungen wie Übelkeit oder Diarrhö (5,6 bzw. 5,0\% der Patienten). Eine C.-difficile-Infektion trat bei sechs Patienten (3\%) in der LevofloxacinGruppe und bei einem Patienten $(0,5 \%)$ in der MoxifloxacinGruppe auf [6].

Besondere Beachtung fanden die möglichen kardiologischen $\mathrm{Ne}$ benwirkungen der beiden Substanzen. Chinolone können - ähnlich wie zahlreiche andere Medikamente aus anderen Wirkstoffgruppen - dosis- bzw. konzentrationsabhängig zu einer QT-Ver- 


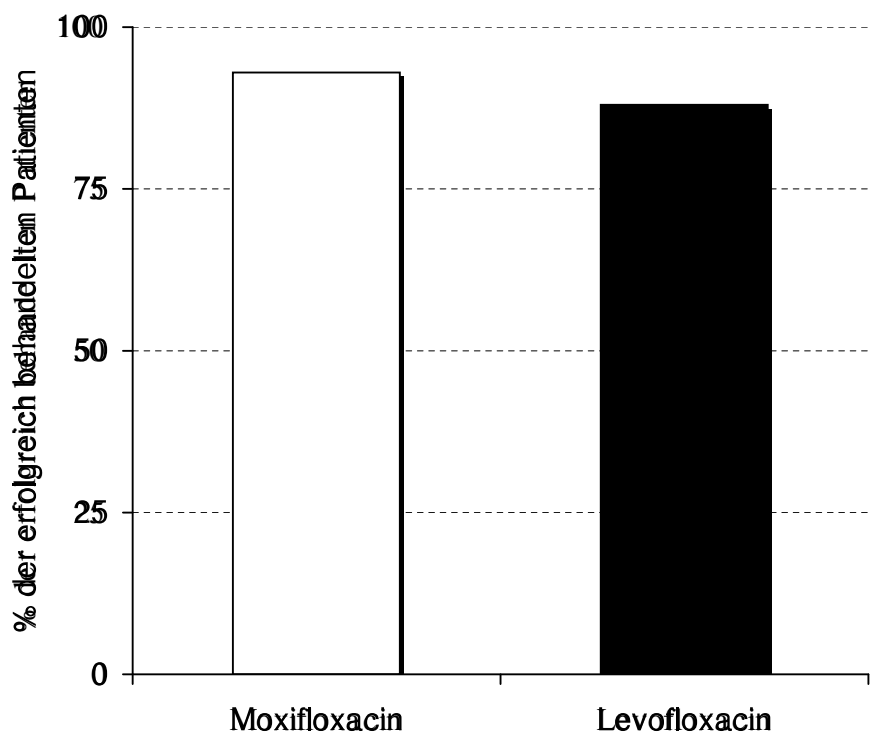

Abb. 2 Wirksamkeitsvergleich von Moxifloxacin und Levofloxacin bei älteren Patienten mit Pneumonie. Jeweils die Hälfte der Patienten erhielt entweder Moxifloxacin $(1 \times \mathrm{tgl} .400 \mathrm{mg})$ oder Levofloxacin $(1 \times \mathrm{tgl}$. $500 \mathrm{mg}$ ) für 7 bis 14 Tage. Beide Präparate wurden für mindestens 2 Tage intravenös verabreicht, anschließend wurde bei entsprechendem klinischen Zustand des Patienten die Therapie mit Tabletten fortgesetzt. Der Therapieerfolg für beide Chinolone lag bei ca. 90\% 5-21 Tage nach Abschluss der Therapie (mod. nach [6]).

längerung führen. Unter therapeutischen Bedingungen ist dieser Effekt minimal und stellt kein Risiko dar. Eine gleichzeitige Anwendung mit anderen Medikamenten, die ein Potenzial zur QTIntervall-Verlängerung aufweisen, sollte nicht erfolgen. Des Weiteren ist es geboten, Moxifloxacin nicht bei Patienten mit angeborenen Störungen der kardialen Reizleitung, bei Patienten mit Elektrolytstörungen (Hypokalimie, Hypomagnesiämie), bei Bradykardie oder anderen schweren kardialen Erkrankungen anzuwenden. Kardiologische Nebenwirkungen wurden in dieser Doppelblindstudie bei 1\% (Moxifloxacin) bzw. 3,5\% (Levofloxacin) der Patienten beobachtet.

\section{Ketolide - Weiterentwicklung der Makrolide}

Makrolide haben sich seit Jahrzehnten zur Behandlung bakterieller Infektionen bewährt. Diese Substanzklasse ist aber auch zunehmend von Resistenzentwicklungen betroffen. Eine Weiterentwicklung der Makrolide sind die Ketolide. Das erste verfügbare Antibiotikum dieser Gruppe ist Telithromycin. Es unterscheidet sich von Erythromycin unter anderem dadurch, dass an Position 3 des Lactonrings nicht der Zucker Cladinose vorkommt, sondern eine Keto-Gruppe. Diese Gruppe verleiht dem Molekül eine hohe Säurestabilität, trägt zur verbesserten antibakteriellen Wirkung bei und stand auch Pate bei der Namensgebung für diese Arzneimittelgruppe.

Telithromycin ist seit etwa 5 Jahren auf dem deutschen Markt erhältlich. Das Antibiotikum wirkt gegen die wichtigsten bakteriellen Erreger von Infektionen der Atemwege. Von klinischer Bedeutung ist die Tatsache, dass auch solche Pneumokokken-Stämme erfasst werden, die gegen Makrolide und $\beta$-Laktamantibiotika resistent sind. Da es bisher keine Zubereitung zur intra- venösen Infusion gibt, sind die therapeutischen Möglichkeiten bei schweren Infektionen begrenzt.

Ähnlich wie die Makrolide, Lincosamide und Streptogramin Typ B (MLSB) hemmen die Ketolide die bakterielle Proteinsynthese infolge der Bindung an die 50S-Untereinheit der Ribosomen. Ketolide interferieren mit der 23S-RNA dieser Untereinheit durch Bindung an zwei Stellen: den Domänen II und V. Darüber hinaus besteht eine direkte Wirkung auf die Bildung der 50S- und 30S-Untereinheiten der Ribosomen. Es ist von therapeutischer Bedeutung, dass Ketolide auch gegen Makrolid-resistente Stämme mit einer induzierbaren MLSB-Resistenz wirken. Außerdem sind sie gegen Stämme wirksam, die durch einen Effluxmechanismus resistent geworden sind.

Telithromycin wirkt konzentrationsabhängig bakterizid gegen Pneumokokken. Ein postantibiotischer Effekt ist nachweisbar. Im Vergleich zu den Makroliden zeigt das Ketolid insbesondere gegen grampositive Erreger (S. pyogenes, S. pneumoniae) eine verbesserte Wirkung. Telithromycin ist auch gegen Erythromycin- und Penicillin-resistente Pneumokokken-Stämme gut wirksam. Es wirkt schließlich auch gegen Staphylokokken, die eine induzierbare MLSB-Resistenz aufweisen, nicht jedoch gegen Staphylokokken mit konstitutiver MLSB-Resistenz [7,8]. Unter den gramnegativen Erregern ist vor allem die Aktivität von Telithromycin gegen Haemophilus influenzae von Interesse: sie entspricht der von Azithromycin; Telithromycin ist damit deutlich wirksamer als Clarithromycin oder Erythromycin gegen Hämophilus. Gegen einen weiteren gramnegativen Erreger von Atemwegsinfektionen, M. catarrhalis, weisen Ketolide und Makrolide eine vergleichbare Aktivität auf. Auch Chlamydien, Mykoplasmen und Legionellen werden erfasst $[7,9]$.

Nach oraler Gabe einer Dosis von $800 \mathrm{mg}$ Telithromycin werden maximale Plasmakonzentrationen von etwa 2 bis $3,5 \mathrm{mg} / \mathrm{l} \mathrm{er-}$ reicht. Die Bioverfügbarkeit liegt bei $57 \%$, die Resorption ist unabhängig von der Nahrungsaufnahme. Die terminale Plasmahalbwertzeit beträgt 10 bis $14 \mathrm{~h}$. Zwischen jungen und älteren Probanden wurden geringe Unterschiede im pharmakokinetischen Verhalten der Substanz gesehen - die Spitzenkonzentrationen im Plasma waren bei älteren Patienten (mittleres Alter 74 Jahre) höher und die Halbwertzeit länger als bei jungen Probanden. Telithromycin wird zu mehreren Metaboliten abgebaut, die in erster Linie mit den Fäzes eliminiert werden. Im Urin wurden weniger als $20 \%$ der Substanz wiedergefunden [7]. Das Antibiotikum reichert sich in der Bronchialschleimhaut, im Alveolarfilm der Lunge („epithelial lining fluid“; ELF) sowie in Alveolarmakrophagen an - damit ist auch eine wichtige Voraussetzung für die Wirkung gegen intrazellulär lokalisierte Erreger gegeben [10].

Nach den bisherigen Ergebnissen der Studien, in denen die Wirksamkeit von Telithromycin nach mikrobiologischen und klinischen Kriterien ausgewertet wurde, kommt das Ketolid vor allem für die Therapie von leichten bis mittelschweren Atemwegsinfektionen infrage. Bei akuter Exazerbation einer chronischen Bronchitis, akuter Sinusitis sowie der Tonsillitis/Pharyngitis ist eine Therapie über 5 Tage, bei ambulant erworbener Pneumonie über 7-10 Tage ausreichend. Zum Vergleich wurden Standardantibiotika wie Clarithromycin oder Amoxicillin eingesetzt. In al- 
len Studien zeigte sich eine gleich gute oder geringfügig bessere Wirksamkeit.

So wurde zum Beispiel in einer Doppelblindstudie die Wirksamkeit von Telithromycin $(1 \times 800 \mathrm{mg})$ mit der von Amoxicillin ( $3 \times$ täglich $1000 \mathrm{mg}$ ) bei 404 Patienten mit ambulant erworbener Pneumonie verglichen. Die nach klinischen Kriterien beurteilte Erfolgsrate war mit 95\% in der Telithromycin-Gruppe tendenziell besser als in der Amoxicillin-Gruppe mit 90\%, bei einer Nachuntersuchung nach etwa 5 Wochen unterschieden sich die Erfolgsraten signifikant (81,4\% vs. $72,7 \%)$ [11].

Telithromycin erwies sich in den klinischen Studien als insgesamt gut verträglich. Die Nebenwirkungen betrafen überwiegend den Gastrointestinaltrakt, dabei waren Diarrhöen und Übelkeit am häufigsten. Eine Erhöhung der Transaminasen als Ausdruck einer Leberfunktionsstörung kann vorkommen. In sehr seltenen Fällen ist ein akutes Leberversagen im Zusammenhang mit einer Telithromycin-Behandlung beschrieben worden.

Telithromycin ist ein Hemmstoff der Cytochrom-abhängigen Monooxygenasen CYP3A4 und CYP2D6. Die Substanz darf daher nicht gleichzeitig mit anderen Arzneimitteln angewandt werden, die über diese Enzyme verstoffwechselt werden. Die Spiegel von Simvastatin steigen zum Beispiel um ein Vielfaches an, wenn Telithromycin gleichzeitig gegeben wird. Während der Behandlung mit Telithromycin müssen daher die Statine vorübergehend abgesetzt werden. Ähnliche Interaktionen können mit Antihistaminika, Benzodiazepinen oder Immunsuppressiva, wie Ciclosporin auftreten. Auch die Plasmaspiegel von Digoxin waren bei gleichzeitiger Gabe erhöht. Ähnliches gilt für Levonorgestrel; die Wirksamkeit von oralen Kontrazeptiva wird jedoch nicht beeinträchtigt. Klinisch relevante Interaktionen mit Theophyllin in retardierter Form wurden nicht beobachtet.

\section{Cethromycin - ein neues orales Ketolid mit ungewisser} Zukunft

Ebenfalls zur Gruppe der Ketolide gehört Cethromycin (ABT-773), das sich noch in der klinischen Entwicklung befindet. Cethromycin zeigt vergleichbar mit Telithromycin eine sehr gute Wirksamkeit gegenüber grampositiven Erregern einschließlich Penicillin- und Erythromycin-resistenten S. pneumoniae-Stämmen, Mykoplasmen und Chlamydien [12]. Die Wirksamkeit gegenüber Haemophilus influenzae ist mit der von Makroliden vergleichbar. Cethromycin verfügt ähnlich wie Telithromycin über einen im Vergleich zu Erythromycin verlängerten postantibiotischen Effekt, was durch die höhere Bindungsaffinität an der 50S Untereinheit der Ribosomen zustande kommt [13].

Die Absorption von Cethromycin im Darm ist dosisabhängig. Bei gesunden Probanden wurden Spitzenwerte im Plasma von im Mittel 0,2 mg/L nach einer Dosis von $150 \mathrm{mg}$ und 0,5 mg/L nach einer Dosis von $300 \mathrm{mg}$ gemessen [14]. Das scheinbare Verteilungsvolumen von Cethromycin ist groß und entspricht dem anderer Ketolide und Makrolide. Hohe intrazelluläre Konzentrationen werden auch in Leukozyten erreicht [15]. Aufgrund seiner guten Penetration in Alveolarzellen und im Alveolarfilm sowie der langen intrapulmonalen Halbwertszeit (bis zu 12 Stunden) dürfte zur antibakteriellen Therapie eine einmal tägliche Gabe ausreichend sein [14]. Bezüglich pharmakokinetischer Interaktionen mit anderen Arzneimitteln treffen aufgrund des Metabolismus über Cytochrom P450-abhängige Monooxygenasen ähnliche Einschränkungen wie bei Telithromycin zu.

In initialen klinischen Studien war Cethromycin gut wirksam und verträglich [16]. Da die Herstellerfirma Abbott die Entwicklung antibakterieller Wirkstoffe nicht mehr vorantreibt, wurde bisher auch die klinische Prüfung von Cethromycin nicht fortgeführt.

\section{Azalide - Makrolidderivate mit reduziertem Interaktionspotenzial}

Seit mehr als 10 Jahren haben sich Azalide bei der Behandlung bakterieller Infektionen der Atemwege in der Praxis bewährt. Sie gehören zu einer Gruppe von Antibiotika, die durch semisynthetische Abwandlung von Makroliden hergestellt werden. Azithromycin, der Wirkstoff von Zithromax ${ }^{\circledR}$, ist bisher der erste und einzige Vertreter der Azalide und wird seit mehr als 10 Jahren zur oralen antibiotischen Therapie angewandt. Das einfache Dosierungsregime ist patientenfreundlich und bedingt eine hohe Compliance. Die seit Anfang 2005 in Deutschland neu verfügbare Zubereitung zur intravenösen Verabreichung erweitert die therapeutischen Möglichkeiten mit diesem Antibiotikum.

Azithromycin erfasst eine Vielzahl aerober und anaerober grampositiver und gramnegativer Erreger. Im grampositiven Bereich werden zum Beispiel Pneumokokken ohne Makrolid-Resistenz zuverlässig erfasst. Im Vergleich zu Erythromycin besteht eine höhere Aktivität im gramnegativen Bereich, z. B. gegenüber Salmonellen, Shigellen, Yersinien, Neisseria gonorrhoeae und Moraxella catarrhalis. Unter therapeutischen Gesichtspunkten ist die wesentlich höhere In-vitro-Aktivität gegenüber Haemophilus influenzae am bedeutsamsten. $\mathrm{Zu}$ den weiteren, therapeutisch relevanten Erregern, die gegenüber Azithromycin empfindlich sind, gehören Campylobacter Spezies, Borrelia burgdorferi, Mycobacterium avium intracellulare und Ureaplasma urealyticum. Durch die im Vergleich zu Erythromycin wesentlich höheren intrazellulären Konzentrationen des Azithromycins ist von einer verbesserten Wirksamkeit gegenüber zellwandlosen und intrazellulär gelagerten Erregern wie Mykoplasmen, Legionellen und Chlamydien auszugehen $[17,18]$.

Im Vergleich zur oralen Formulierung lassen sich mit intravenös verabreichtem Azithromycin deutlich höhere Konzentrationen erzielen. Im direkten „Cross-over“-Vergleich wurden bei Probanden nach einer Einzeldosis von $500 \mathrm{mg}$ AUC-Werte (0 bis 72 Stunden) von $3,39 \mathrm{mg} / \mathrm{l} \times \mathrm{h}$ nach oraler Gabe und $9,08 \mathrm{mg} / \mathrm{l} \times \mathrm{h}$ nach intravenöser Gabe errechnet. Bei mehrmaliger i.v.-Applikation war nach der fünften Dosis die Spitzenkonzentration im Serum mit etwa $1,1 \mathrm{mg} / \mathrm{l}$ praktisch unverändert geblieben, umso deutlicher zeigte sich eine Vergrößerung der AUC um etwa $60 \%$. Die Eliminationshalbwertzeit von Azithromycin hängt vom Bestimmungszeitpunkt ab. Im Intervall von 8-24 Stunden nach i.v.-Verabreichung einer $500 \mathrm{mg}$-Dosis beträgt sie 11 - 14 Stunden und verlängert sich im Bestimmungszeitraum von 24-72 Stunden nach der Infusion auf 35-40 Stunden. Azithromycin wird vorwiegend durch biliäre Exkretion eliminiert [17,19-21]. 
Azithromycin besitzt eine hohe Gewebeaffinität, die durch ein zusätzliches basisches Stickstoffatom im Molekül zu erklären ist. Für die klinische Wirksamkeitsvorhersage sind daher nicht die Serumkonzentrationen, sondern die Gewebekonzentrationen relevant, die durch intrazelluläre Anreicherung und Phagozytentransport an den Infektionsort erreicht werden [22].

Mit der intravenösen Zubereitung des Azithromycins können vor allem schwere ambulant erworbene Pneumonien behandelt werden (in Kombination mit einem Betalaktamantibiotikum oder Carbapenem). Der intravenösen Therapie sollte immer eine orale Azithromycin-Gabe folgen. Die Dosis von Azithromycin i.v. beträgt $500 \mathrm{mg}$ über mindestens ein bis zwei Tage, gefolgt von täglichen oralen Dosen von 250 oder $500 \mathrm{mg}$ zur Vervollständigung einer sieben- bis zehntägigen Behandlungsperiode. Die empfohlene Konzentration der Infusionslösung und die Infusionsdauer betragen $1 \mathrm{mg} / \mathrm{ml}$ über drei Stunden.

Die häufigste unerwünschte Wirkung von Makroliden und verwandten Antibiotika sind gastrointestinale Störungen, die durch eine Stimulierung der Motilin-Rezeptoren hervorgerufen werden. Die gastrointestinale Intoleranz kann sehr ausgeprägt und Therapie-limitierend sein. Die langjährige Erfahrung mit den säurestabilen Derivaten Clarithromycin, Roxithromycin oder Azithromycin zeigt, dass gastrointestinale Nebenwirkungen seltener auftreten als nach Erythromycin; dies gilt auch für die intravenösen Zubereitungen. Neben der gastrointestinalen Verträglichkeit wurde auch die lokale Verträglichkeit von infundiertem Azithromycin, Clarithromycin und Erythromycin im direkten Vergleich überprüft (Abb. 3) [23].

Im Gegensatz zu den Makroliden/Ketoliden führt Azithromycin nicht zu einer Hemmung der Cytochrom-P450-abhängigen Monooxygenasen. Das Risiko für Interaktionen mit gleichzeitig verabreichten Arzneimitteln ist daher gering (Abb.4).

Oxazolidinone - neue Therapieoption bei Infektionen durch grampositive Erreger

Die Entdeckung der Oxazolidinone begann bereits 1978, als in einer Patentschrift für DuPont Verbindungen beschrieben wurden, die primär gegen mikrobielle Erkrankungen von Pflanzen vorgesehen waren. Im Jahre 1987 wurden die Oxazolidinone als eine neue Klasse synthetischer Wirkstoffe zur Anwendung in der Humanmedizin vorgestellt. Die antibakterielle Aktivität erstreckt sich vor allem gegen grampositive Erreger. Die wirksamste Substanz wurde bei der Firma DuPont aufgrund schwerwiegender Toxizität jedoch nicht weiterentwickelt, obwohl es in vitro und auch in Tiermodellen gut wirksam war. Das erste in der Humanmedizin verwendbare Oxazolidinon ist Linezolid, das seit 2001 in Deutschland unter dem Namen Zyvoxid $^{\circledR}$ zur Verfügung steht.

Linezolid entfaltet seine antibakterielle Wirkung durch Hemmung der bakteriellen Proteinsynthese [24]. Es bindet - ähnlich wie Chloramphenicol - an die 50S-Untereinheit der bakteriellen Ribosomen, verhindert die Bildung eines funktionstüchtigen Initiationskomplexes und interferiert so mit einem frühen Schritt der Proteinbiosynthese. Im Gegensatz zu Chloramphenicol wir-

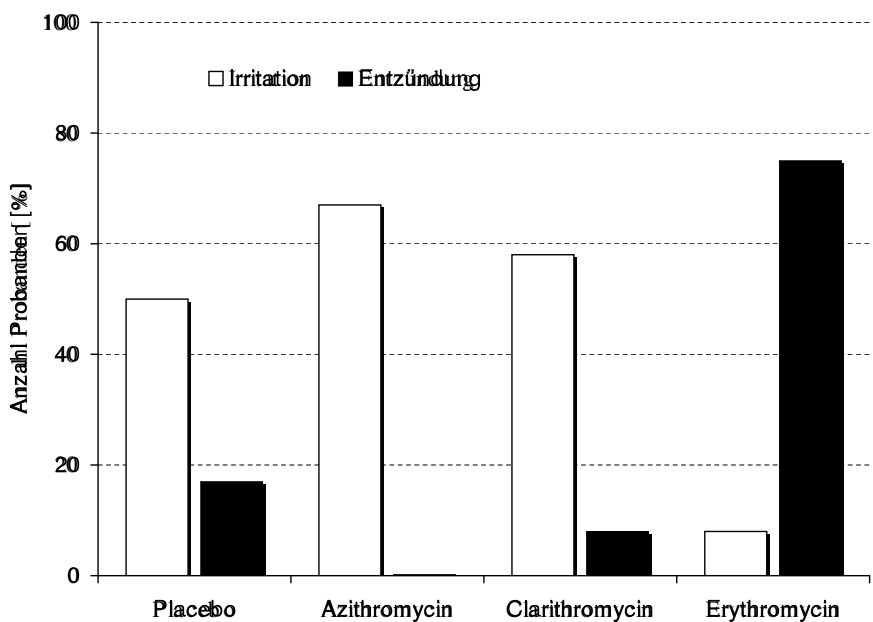

Abb. 3 Lokale Unverträglichkeitsreaktion bei intravenöser Behandlung mit Makroliden/Azaliden im Vergleich zu Plazebo. 12 Probanden wurden 3 Tage lang behandelt mit: $3 \times$ Plazebo (in $500 \mathrm{ml} 0.9 \% \mathrm{NaCl}-$ Lsg.), $1 \times 500 \mathrm{mg}$ Azithromycin Dihydrat (in $250 \mathrm{ml} 0,9 \% \mathrm{NaCl}$-Lsg.), $2 \times 500 \mathrm{mg}$ Clarithromycin Lactobionat (in $250 \mathrm{ml} 0,9 \% \mathrm{NaCl}$-Lsg.) und $3 \times 500 \mathrm{mg}$ Erythromycin Lactobionat (in $250 \mathrm{ml} \mathrm{0,9 \%} \mathrm{NaCl-Lsg.);} \mathrm{Infu-}$ sionsdauer: 1 Stunde. Die häufigste lokale Reaktion war ein „Irritationssyndrom", gefolgt von Schmerzen, Inflammation und Phlebitis. Phlebitis wurde ausschließlich nach Infusion der Clarithromycin-Zubereitung beobachtet (bei 6 von 12 Probanden). Schmerzen an der Infusionsstelle wurden von allen 12 Studienteilnehmern nach Clarithromycin, von 7 von 12 Teilnehmern (58\%) nach Azithromycin und 3 von 12 Teilnehmern (25\%) nach Erythromycin angegeben. Nur von einem Studienteilnehmer, der Plazebo-Lösung erhielt, wurden Schmerzen angegeben (mod. n. [23]).

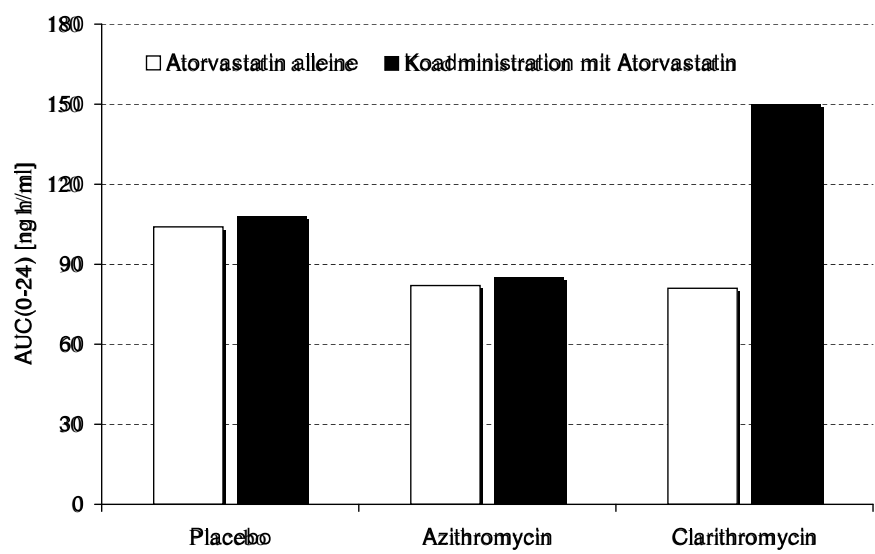

Abb. 4 Interaktionspotenzial von Azithromycin und Clarithromycin mit Atorvastatin.

Im Gegensatz zu Clarithromycin führt die Einnahme von Azithromycin bei gleichzeitiger Gabe von Atorvastatin nicht zu einer Erhöhung der Plasma AUC-Werte von Atorvastatin, weil Azithromycin nicht mit dem Cytochrom P450-abhängigen Stoffwechselweg des Lipidsenkers interferiert (mod. nach [43]).

ken Oxazolidinone nicht durch Hemmung der Peptidyltransferase. Die therapeutisch relevante Aktivität von Linezolid beschränkt sich auf grampositive Erreger. Die meisten Streptokokken, Staphylokokken und Enterokokken werden durch Konzentrationen von $<4 \mathrm{mg} / \mathrm{l}$ erfasst. Die antibakterielle Aktivität ist unabhängig davon, ob die geprüften Stämme resistent gegen Penicillin, Oxacillin oder Vancomycin sind. H. influenzae, ein bedeutsamer gramnegativer Erreger von Infektionen der Atemwege, wird erst bei Konzentrationen von $4-16 \mathrm{mg} / \mathrm{l}$ gehemmt und 
muss daher als überwiegend resistent eingestuft werden. Enterobacteriaceae und Pseudomonas-Stämme weisen eine natürliche Resistenz auf (MHK > 64 mg/l) [25].

Nach oraler Gabe wird Linezolid vollständig resorbiert. Etwa 1 bis 2 Stunden nach der Einnahme werden Spitzenkonzentrationen gemessen, die nach mehrfacher Gabe von $600 \mathrm{mg}$ (alle 12 Stunden) zwischen 6 und $21 \mathrm{mg} / \mathrm{l}$ (Tal- und Spitzenkonzentrationen) liegen [24]. Die Resorption wird durch gleichzeitige Nahrungsaufnahme nicht relevant beeinflusst. Die Plasmaproteinbindung von Linezolid liegt bei $30 \%$, das Verteilungsvolumen der Substanz wurde mit etwa 0,6 bis $0,7 \mathrm{l} / \mathrm{kg}$ berechnet. Linezolid wird zu zwei Hauptmetaboliten abgebaut, die dann neben der unveränderten Substanz im Urin eliminiert werden. Die Eliminationshalbwertzeit liegt zwischen 5 und 7 Stunden. CytochromP450-abhängige Monooxygenasen werden offenbar weder gehemmt noch induziert. Bei Patienten mit renaler Insuffizienz kommt es nicht zum Anstieg der Arzneimittelkonzentrationen im Blut. Allerdings ist noch unklar, ob sich Risiken durch eine Kumulation der Hauptmetaboliten bei Patienten mit ausgeprägter Niereninsuffizienz ergeben könnten [23,25,27].

Anwendungsgebiete für Linezolid sind sowohl die ambulant als auch die im Krankenhaus erworbene Pneumonie durch grampositive Erreger, darunter multiresistente Keime [28], sowie schwere Hautinfektionen durch Staphylokokken oder Streptokokken. Die Indikation für eine Behandlung mit dem Oxazolidinon sollte streng gestellt werden, da - wie bei allen Antibiotika - befürchtet werden muss, dass eine breite Anwendung zu einer raschen Ausbreitung resistenter Stämme führt [27].

Während der klinischen Prüfung war die Verträglichkeit von Linezolid insgesamt ähnlich wie die der vergleichend untersuchten Antibiotika. Gastrointestinale Störungen und leichte ZNSSymptome standen im Vordergrund. Erbrechen trat unter Linezolid bei 3,7\% der Patienten auf, in der Vergleichsgruppe dagegen nur bei 2,0\% der Behandelten (etwa 2000 Patienten pro Gruppe); bei 1,2\% bzw. 0,4\% wurde dies als Arzneimittel-assoziiert beurteilt. Bei längerer Behandlungsdauer ( $>2$ Wochen) wurden mit Linezolid Blutbildveränderungen gesehen [29]. Bei Patienten mit einer vorbestehenden Thrombozytopenie oder bei Gabe anderer Medikamente, die eine Thrombozytopenie verursachen können, sollten daher während der Behandlung mit Linezolid genaue Blutbildkontrollen durchgeführt werden; eine wöchentliche Blutbildkontrolle ist generell angezeigt. Bei Patienten, die mit Zyvoxid behandelt wurden, sind periphere und/oder optische Neuropathien berichtet worden, teilweise mit nachfolgender Erblindung. Diese Meldungen stammen überwiegend von Patienten, die über einen längeren Zeitraum als die maximal empfohlenen 28 Tage behandelt wurden.

Linezolid ist ein Hemmstoff der Monaminoxidase. Entsprechende Interaktionen mit gleichzeitig gegebenen adrenerg oder serotonerg wirksamen Medikamenten können daher auftreten. Bei einer gleichzeitigen Einnahme von „Erkältungsmedikamenten“, die zum Beispiel Pseudoephedrin enthalten können, ist deshalb der Blutdruck zu kontrollieren, da die Kombination mit Linezolid zum Blutdruckanstieg führt. Die Patienten sollten auch darauf hingewiesen werden, dass während der Behandlung übermäßige Mengen von Nahrungsmitteln oder Getränken mit hohem Gehalt an Tyramin (z.B. Käse, Rotwein etc.) vermieden werden müssen $[27,30]$.

Carbapeneme - $\beta$-Laktamantibiotilka mit breitem Spektrum

Bei den $\beta$-Laktam-Antibiotika steht mit Ertapenem (Invanz ${ }^{\circledR}$ ) seit 2003 in Deutschland ein weiteres Antibiotikum aus dieser Gruppe zur Verfügung. Die bisher verfügbaren Carbapeneme, Imipenem und Meropenem, haben sich seit ihrer Einführung als wichtige Reserveantibiotika zur stationären, parenteralen Therapie schwerer Infektionen bewährt. Carbapeneme zeichnen sich durch ein breites antibakterielles Spektrum aus. Ertapenem eignet sich zur parenteralen Behandlung schwerer Infektionen durch gramnegative oder grampositive Erreger, einschließlich Anaerobier [31].

Als $\beta$-Laktam-Antibiotikum hemmt Ertapenem die Synthese der Zellwand von Bakterien durch Bindung an Penicillin-bindende Proteine (PBP). Zumindest bei E. coli ist die Affinität zu den PBP 2 und 3 am höchsten. Es wirkt, wie die anderen Carbapeneme, gegen ein breites Spektrum von gramnegativen und grampositiven Erregern sowie gegen Anaerobier einschließlich B. fragilis. Im direkten Vergleich mit Imipenem erwies sich Ertapenem als wirksamer gegen gramnegative Bakterien, war aber etwas schwächer wirksam gegen Streptokokken, Staphylokokken und Anaerobier. Die minimalen Hemmkonzentrationen lagen jedoch höchstens bei $0,5 \mathrm{mg} / \mathrm{l}$ und weisen das Antibiotikum damit als sehr wirksam aus. Ertapenem ist klinisch ohne relevante Aktivität gegenüber P. aeruginosa [32-34].

Ertapenem wird einmal täglich in einer Dosis von 1,0 g intravenös infundiert. Am Ende einer 30-minütigen Infusion von $1 \mathrm{~g} \mathrm{Er}$ tapenem liegen die mittleren Plasmakonzentrationen bei gesunden Erwachsenen bei $155 \mathrm{mg} / \mathrm{l}$. Die Eliminationshalbwertzeit beträgt etwa 4 Stunden und ist damit deutlich länger als bei den bisher bekannten Carbapenemen. Die Proteinbindung der Substanz ist mit 92 bis $95 \%$ relativ hoch, das Verteilungsvolumen wurde mit 8 Litern berechnet. Die Ausscheidung erfolgt überwiegend renal, im Urin wird die Substanz etwa zu gleichen Anteilen als unveränderte Substanz oder in Form eines Metaboliten gefunden. Die Metabolisierung besteht in einer Dehydropeptidase-vermittelten Hydrolyse des $\beta$-Laktamringes.

Bei Patienten mit leichter Niereninsuffizienz (Kreatinin-Clearance $31-59 \mathrm{ml} / \mathrm{min} / 1,73 \mathrm{~m}^{2}$ ) sind die AUC-Werte im Vergleich zu gesunden Erwachsenen etwa 1,5 bis 1,8mal größer; diese Veränderung der Kinetik erfordert keine Dosisanpassung. Ausreichende Daten, die eine Dosierungsempfehlung bei Patienten mit fortgeschrittener Niereninsuffizienz ermöglichen würden, liegen zurzeit noch nicht vor. Das gleiche gilt für Hämodialyse-Patienten und Patienten mit Leberinsuffizienz [35].

Ertapenem ist zur Behandlung der folgenden Infektionen zugelassen, wenn diese durch empfindliche Erreger verursacht sind: (1) intraabdominelle Infektionen, (2) ambulant erworbene Pneumonie und (3) akute gynäkologische Infektionen. Die Gleichwertigkeit des Carbapenems mit Standardtherapeutika wurde bei diesen Indikationen während der klinischen Prüfung nachgewiesen. 
In einer umfangreichen Doppelblind-Studie an mehr als $500 \mathrm{~Pa}-$ tienten mit ambulant erworbener Pneumonie wurde Ertapenem mit Ceftriaxon verglichen. Beide Antibiotika wurden einmal täglich in einer Dosierung von 1,0 g intravenös verabreicht; die Dauer der intravenösen Behandlung lag bei etwa 4 Tagen (Medianwert). Anschließend wurden die meisten Patienten oral mit Coamoxiclav weiterbehandelt. Die Therapie zeigte in beiden Gruppen einen gleich guten klinischen Erfolg bei mehr als 90\% der Patienten in beiden Gruppen. Auch hinsichtlich der Verträglichkeit ergaben sich keine Unterschiede [36].

Ertapenem erwies sich in der klinischen Prüfung als gut verträglich. Am häufigsten waren Diarrhö (4,8\%), Übelkeit (2,8\%) und Komplikationen an der Infusionsstelle (4,5\%). Auch Haut-Reaktionen (Exantheme, Juckreiz) und ZNS-Reaktionen, wie Kopfschmerzen, Verwirrtheitszustände und Krampfanfälle, wurden während der Behandlung beobachtet. Nur bei 1,3\% der Patienten musste die Therapie aufgrund von unerwünschten Wirkungen abgebrochen werden.

Wechselwirkungen zwischen Ertapenem und anderen gleichzeitig verabreichten Arzneimitteln sind bisher nicht bekannt. Da Carbapeneme die Serum-Konzentrationen von Valproinsäure senken können, wird eine Spiegelbestimmung des Antiepileptikums empfohlen, wenn beide Medikamente gleichzeitig gegeben werden sollen.

\section{Neues Carbapenem - Doripenem}

Ein neues vollsynthetisches Carbapenem ist Doripenem, das sich noch in der klinischen Entwicklung befindet. Es ist aufgrund seiner breiten antibakteriellen Wirkung zur Behandlung von hospitalisierten Patienten mit schweren bakteriellen Infektionen geeignet. Doripenem zeigte in mehreren in-vitro Studien eine ähnliche Aktivität wie Meropenem gegenüber gramnegativen Erregern bzw. Imipenem gegenüber gramnegativen Bakterien. Wie alle Carbapeneme ist Doripenem gegen Enterobacteriaceae mit Carbapenemase-Aktivität wirkungslos. Das Antibiotikum ist in vitro sehr gut wirksam gegen $\mathrm{H}$. influenza, Moraxella catarrrhalis, Aeromonas spp., Bacillus spp., Bordetella pertussis und andere häufig vorkommende grampositive wie gramnegative Erreger. Die Aktivität gegenüber P. aeruginosa entspricht der von Meropenem bzw. ist stärker im Vergleich zu Imipenem [37].

Bisher sind erst wenige klinische Daten zu Doripenem veröffentlicht worden. Ca. 60 - 75\% einer Dosis von 1,0 g wurden unverändert über die Niere ausgeschieden; die maximale Plasmakonzentration betrug $50 \mu \mathrm{g} / \mathrm{ml}$ nach einer einstündigen Infusion. Unerwünschte Ereignisse wie renale Toxizität und ZNS-Nebenwirkungen scheinen bei Doripenem im Vergleich zu Imipenem und Meropenem deutlich weniger häufig aufzutreten. Doripenem ist daher ein interessanter Kandidat für ein weiteres Breitspektrum-Antibiotikum aus dieser Gruppe.
Lipopeptide - Inaktivierung durch Surfactant

Zu den kürzlich neu in den USA eingeführten Antiinfektiva gehört Daptomycin, ein Lipopeptid mit einem neuartigen Wirkmechanismus. Daptomycin ist dort zur Behandlung schwerer Hautund Weichteilinfektionen zugelassen und steht ausschließlich zur intravenösen Applikation zur Verfügung.

Daptomycin ist u.a. sehr gut wirksam gegen Methicillin-resistente S. aureus-Stämme und Glycopeptid-resistente Enterokokken. Die Wirkung erklärt sich durch eine Kalzium-abhängige Insertion in die Zytoplasmamembran, die zu einem Verlust des Membranpotenzials durch Efflux von Kalium und in der Folge durch eine deregulierte Biosyntheseleistung zum raschen Zelltod führt. Daptomycin bietet neben seiner schnellen bakteriziden Wirkung den Vorteil, dass es auch in der stationären Phase seine bakterizide Wirkung aufrechterhält. Ein post-antibiotischer Effekt von 1- 6 Stunden ist beschrieben worden. „Problemkeime“" werden bei Konzentrationen $<4 \mathrm{mg} / \mathrm{l}$ erfasst [38].

Die klinische Besonderheit von Daptomycin besteht darin, dass das Molekül mit Surfactant interagiert und dadurch in seiner antibakteriellen Aktivität negativ beeinflusst wird. Obwohl Daptomycin in vitro sehr gut wirksam gegen $S$. pneumoniae ist $\left(\mathrm{MHK}_{90}: 0,06 \mathrm{mg} / \mathrm{l}\right)$, konnte in Phase-III Studien keine Gleichwertigkeit im Vergleich mit der Gabe von Ceftriaxon gezeigt werden (Wirksamkeit 79\% für Daptomycin und 87\% für Ceftriaxon). Erst eine genauere Analyse dieser Ergebnisse und gezielte in vitroVersuche ließen die organspezifische Hemmung dieses Wirkprinzips erkennen. Daptomycin ist ein gutes Beispiel dafür, dass neben der antibakteriellen Potenz sowie der Fähigkeit zur Penetration in Lungengewebe, Alveloarfilm und Alveolarmakrophagen auch die Besonderheiten der pulmonalen Physiologie für die antibiotische Funktion berücksichtigt werden müssen [39].

\section{Glycylcycline - neue Tetracyclin-Derivate}

Ein weiteres Antibiotikum, das derzeit nur auf dem US-amerikanischen Markt verfügbar ist, heißt Tigecyclin $\left(\right.$ Tygacil $\left.^{\circledR}\right)$. Es wurde 2005 von der FDA in einem beschleunigten Verfahren zugelassen. Mit der Zulassung in Europa wird im Laufe des Jahres 2006 gerechnet. Das Glycylcyclin-Antibiotikum zeichnet sich durch eine Glycylamidogruppe an Position 9 von Minocyclin aus und weist ein breites antibakterielles Spektrum auf. Die Substanz ist wirksam bei Infektionen, die durch gramnegative und grampositive Bakterien, Anaerobier und Methicillin-empfindliche sowie Methicillin-resistente S. aureus-Stämme hervorgerufen werden. Tigecyclin wurde bei entsprechendem Erregernachweis zunächst zur Behandlung schwerer Haut- und Weichteilinfektionen sowie bei komplizierten abdominellen Infektionen zugelassen und steht bisher nur zur intravenösen Infusion zur Verfügung. Durch die gute Wirksamkeit bei multiresistenten grampositiven Erregern ist die Substanz auch bei Pneumokokkeninfektionen interessant. Die Untersuchungen zur Wirksamkeit und Verträglichkeit bei ambulant und nosokomial erworbenen Pneumonien sind noch nicht abgeschlossen. Derzeit wird in den USA eine große multizentrische Phase III-Studie mit 430 Patienten durchgeführt, die Tigecyclin mit Imipenem/Cilastatin bei Patienten mit nosokomial erworbener bakterieller 
Pneumonie vergleicht. Weitere Erfahrungen mit dem neuen Antibiotikum sind notwendig, um den genauen therapeutischen Stellenwert besser definieren zu können.

Tigecyclin bindet an die 30 s Untereinheiten der bakteriellen Ribosomen und verhindert den Übertritt der Aminoacyl-tRNS-Moleküle auf die A-Seite der Ribosomen. Diese Blockade verhindert das Einfügen von Aminosäuren in die sich verlängernde Proteinkette. Die Einzigartigkeit seiner chemischen Substitutionsgruppe, die man weder bei natürlichen noch bei semi-synthetischen Tetracyclinen findet, verleiht Tigecyclin seine mikrobiologischen Qualitäten. Das Antibiotikum umgeht zwei bedeutende Resistenzmechanismen - Effluxpumpen und ribosomale Schutzmechanismen -, die den Einsatz vieler Antibiotika einschränken.

Die Pharmakokinetik von Tigecyclin wurde an gesunden Probanden untersucht. Die Substanz zeigt eine lineare Pharmakokinetik und verfügt über eine lange terminale Halbwertszeit, so dass das Fließgleichgewicht nach etwa 7 Tagen erreicht wird. Tigecyclin verteilt sich in hohem Maße im Gewebe. Die Pharmakokinetik des Glycylcyclin-Antibiotikums wird durch Alter, eingeschränkte Nierenfunktion und Nahrungsaufnahme nicht wesentlich beeinflusst. Bei dialysepflichtigen Patienten ist ebenfalls keine Dosisanpassung erforderlich. Bei Patienten mit höhergradiger Leberfunktionsstörung (Child Pugh C) sollte die Erhaltungsdosis reduziert werden. Die empfohlene Infusionsdauer beträgt 30-60 min. Tigecyclin wird nur unvollständig metabolisiert und zu einem großen Teil unverändert eliminiert. Interaktionen mit anderen Medikamenten, die über mikrosomale Cytochromabhängige Monooxigenasen verstoffwechselt werden, sind bei fehlender Affinität von Tigecyclin zu diesen Enzymsystemen nicht zu erwarten.

In klinischen Studien war Tigecyclin (50 mg i.v., Dosisintervall 12 Std.) nicht optimal verträglich. Unerwünschte Wirkungen waren hauptsächlich Übelkeit und Erbrechen, die meistens am ersten oder zweiten Tag nach Beginn der Therapie auftraten. Dies war auch am häufigsten der Grund für einen Studienabbruch bei ca. $5 \%$ der Patienten. Es bestand hinsichtlich der Abbruchrate kein Unterschied zwischen Tigecyclin und zum Vergleich angewendeten Antibiotika. Von Übelkeit war in etwa ein Drittel der Behandelten betroffen, Erbrechen kam bei etwa 20\% vor. Die Verträglichkeit kann bei nüchternen Personen durch den Einsatz von Antiemetika verbessert werden. C. difficile-assoziierte Komplikationen (pseudomembranöse Kolitis) mit Tigecyclin sind selten $[40,41]$.

Fazit

Trotz einiger vielversprechender Ansätze und Neueinführungen in den vergangenen Jahren stellt der Innovationsrückgang bei der Entwicklung neuer Antibiotika eine ernst zu nehmende Gefahr für zukünftige Behandlungsstrategien dar. Eine dauerhaft erfolgreiche antibakterielle Therapie muss zwangsläufig die zunehmende Resistenzentwicklung im Auge behalten. Gerade deshalb ist der besonnene Umgang mit neuen, aber auch länger verfügbaren Antibiotika umso wichtiger, denn der Wettlauf mit den Erregern ist nicht allein durch neue Substanzen zu gewinnen.
${ }^{1}$ Bosso JA. The antimicrobial armamentarium: evaluating current and future treatment options. Pharmacotherapy 2005; 25: 55S-62S

2 Talbot Jr GH, Bradley J, Edwards JE et al. Antimicrobial Availability Task Force of the Infectious Diseases Society of America. Bad bugs need drugs: an update on the development pipeline from the Antimicrobial Availability Task Force of the Infectious Diseases Society of America. Clin Infect Dis 2006; 42 (5): 657-668

${ }^{3}$ Stahlmann R. Clinical toxicological aspects of fluoroquinolones. Toxicol Lett 2002; 127: 269-277

${ }^{4}$ NN. Full Prescribing Information for Avelox ${ }^{\circledR}$ IV. Bayer Health Care, 2005

${ }^{5}$ Finch R, Schurmann D, Collins O et al. Randomized controlled trial of sequential intravenous (i.v.) and oral moxifloxacin compared with sequential i.v. and oral co-amoxiclav with or without clarithromycin in patients with community-acquired pneumonia requiring initial parenteral treatment. Antimicrob Agents Chemother 2002; 46: $1746-1754$

${ }^{6}$ Anzueto A, Niederman MS, Pearle J et al. Community-Acquired Pneumonia Recovery in the Elderly Study Group. Community-Acquired Pneumonia Recovery in the Elderly (CAPRIE): efficacy and safety of moxifloxacin therapy versus that of levofloxacin therapy. Clin Infect Dis 2006; $42: 73-81$

${ }^{7}$ Balfour JA, Figgitt DP. Telithromycin. Drugs 2001; 61: 815-829

${ }^{8}$ Jalava J, Kataja J, Seppala $\mathrm{H}$ et al. In vitro activities of the novel ketolide telithromycin (HMR 3647) against erythromycin-resistant Streptococcus species. Antimicrob Agents Chemother 2001; 45: 789-793

${ }^{9}$ Hammerschlag MR, Roblin PM, Bebear CM. Activity of telithromycin, a new ketolide antibacterial, against atypical and intracellular respiratory tract pathogens. J Antimicrob Chemother 2001; 48: 25-31

${ }^{10}$ Khair OA, Andrews JM, Honeybourne D et al. Lung concentrations of telithromycin after oral dosing. J Antimicrob Chemother 2001; 47: $837-840$

${ }^{11}$ Hagberg L, Torres A, Rensburg D van et al. Efficacy and tolerability of once-daily telithromycin compared with high-dose amoxicillin for treatment of community-acquired pneumonia. Infection 2002; 30: $378-386$

12 Reinert RR. Clinical efficacy of ketolides in the treatment of respiratory tract infections. J Antimicrob Chemother 2004; 53: 918-927

${ }^{13}$ Cao Z, Zhong P, Ruan X et al. Ribosome affinity and the prolonged molecular postantibiotic effect of cethromycin (ABT-773) in Haemophilus influenzae. Int J Antimicrob Agents 2004; 24: 362 - 368

${ }^{14}$ Conte Jr JE, Golden JA, Kipps J et al. Steady-state plasma and intrapulmonary pharmacokinetics and pharmacodynamics of cethromycin. Antimicrob Agents Chemother 2004; 48: 3508 - 3515

${ }^{15}$ Garcia I, Pascual A, Ballesta S et al. Accumulation and activity of cethromycin (ABT-773) within human polymorphonuclear leucocytes. J Antimicrob Chemother 2003; 52: 24-28

${ }^{16}$ Zhanel GG, Walters M, Noreddin A et al. The ketolides: a critical review. Drugs 2002; 62: $1771-1804$

${ }^{17}$ NN. Full Prescribing Information for Zithromax ${ }^{\circledR}$ IV. Pfizer, 2002

${ }^{18}$ Peters DH, Friedel HA, McTavish D. Azithromycin. A review of its antimicrobial activity, pharmacokinetic properties and clinical efficacy. Drugs 1992; 44: 750-799

${ }^{19}$ Foulds G, Shepard RM, Johnson RB. The pharmacokinetics of azithromycin in human serum and tissues. J Antimicrob Chemother 1990; 25: $73-82$

${ }^{20}$ Luke DR, Foulds G, Cohen SF et al. Safety, toleration, and pharmacokinetics of intravenous azithromycin. Antimicrob Agents Chemother 1996; 40: 2577-2581

${ }^{21}$ Chiu LM, Menhinick AM, Johnson PW et al. Pharmacokinetics of intravenous azithromycin and ceftriaxone when administered alone and concurrently to healthy volunteers. J Antimicrob Chemother 2002; 50: $1075-1079$

${ }^{22}$ Rodvold KA, Danziger LH, Gotfried MH. Steady-state plasma and bronchopulmonary concentrations of intravenous levofloxacin and azithromycin in healthy adults. Antimicrob Agents Chemother 2003; 47: $2450-2457$

${ }^{23}$ Zimmermann T, Laufen H, Riedel KD et al. Comparative tolerability of intravenous azithromycin, clarithromycin and erythromycin in healthy volunteers: results of a double-blind, double-dummy, four-way crossover study. Clin Drug Invest 2001; 21: 527-536 
${ }^{24}$ Diekema DI, Jones RN. Oxazolidinones: a review. Drugs 2000; 59: 7-16

${ }^{25}$ Clemett D, Markham A. Linezolid. Drugs 2000; 59: 815-827

${ }^{26}$ Gee T, Ellis R, Marshall G et al. Pharmacokinetics and tissue penetration of linezolid following multiple oral doses. Antimicrob Agents Chemother 2001; 45: 1843-1846

${ }^{27}$ NN. Fachinformation zu Zyvoxid ${ }^{\circledR}$. Pharmacia GmbH, 2001

${ }^{28}$ Rubinstein E, Cammarata S, Oliphant T et al. Linezolid (PNU-100766) versus vancomycin in the treatment of hospitalized patients with nosocomial pneumonia: a randomized, double-blind, multicenter study. Clin Infect Dis 2001; 32: $402-412$

${ }^{29}$ Green SL, Maddox JC, Huttenbach ED. Linezolid and reversible myelosuppression. JAMA 2001; 285: 1291

${ }^{30}$ Humphrey SJ, Curry JT, Turman CN et al. Cardiovascular sympathomimetic amine interactions in rats treated with monoamine oxidase inhibitors and the novel oxazolidinone antibiotic linezolid. J Infect Dis 2001; 37: 548-563

${ }^{31}$ Bonfiglio G, Russo G, Nicoletti G. Recent developments in carbapenems. Expert Opin Investig Drugs 2002; 11: 529-544

${ }^{32}$ Livermore DM, Carter MW, Bagel S et al. In vitro activities of ertapenem (MK-0826) against recent clinical bacteria collected in Europe and Australia. Antimicrob Agents Chemother 2001; 45: 1860-1867

${ }^{33}$ Fuchs PC, Barry AL, Brown SD. In vitro activities of ertapenem (MK-0826) against clinical bacterial isolates from 11 North American medical centers. Antimicrob Agents Chemother 2001; 45: 1915 - 1918

${ }^{34}$ Goldstein EJ, Citron DM, Vreni Merriam MC et al. Comparative In vitro activities of ertapenem (MK-0826) against 1,001 anaerobes isolated from human intra-abdominal infections. Antimicrob Agents Chemother 2000; 44: 2389-2394

${ }^{35}$ NN. Fachinformation zu Invanz ${ }^{\circledR}$. MSD Haar, 2005

${ }^{36}$ Ortiz-Ruiz G, Caballero-Lopez J, Friedland IR et al. Protocol 018 Ertapenem Community-Acquired Pneumonia Study Group. A study evaluating the efficacy, safety, and tolerability of ertapenem versus ceftriaxone for the treatment of community-acquired pneumonia in adults. Clin Inf Dis 2002; 34: 1076- 1083

${ }^{37} \mathrm{Ge}$ Y, Wikler MA, Sahm DF et al. In vitro antimicrobial activity of doripenem, a new carbapenem. Antimicrob Agents Chemother 2004; 48: $1384-1396$

${ }^{38}$ NN. Full Prescribing Information for Cubicin ${ }^{\circledR}$. Cubist Pharmaceuticals, 2005

${ }^{39}$ Silverman JA, Mortin LI, Vanpraagh AD et al. Inhibition of daptomycin by pulmonary surfactant: in vitro modeling and clinical impact. J Infect Dis 2005; 191: 2149-2152

${ }^{40}$ NN. Full Prescribing Information for Tygacil ${ }^{\circledR}$. Wyeth Pharmaceuticals, 2005

${ }^{41}$ Rello J. Pharmacokinetics, pharmacodynamics, safety and tolerability of tigecycline. J Chemother 2005; 17: 12-22

${ }^{42}$ Saravolatz LD, Leggett J. Gatifloxacin, gemifloxacin, and moxifloxacin: the role of 3 newer fluoroquinolones. Clin Infect Dis 2003; 37: $1210-1215$

${ }^{43}$ Amsden GW, Kuye O, Wei GC. A study of the interaction potential of azithromycin and clarithromycin with atorvastatin in healthy volunteers. J Clin Pharmacol 2002; 42: 444-449 